\title{
Neutrino physics with particle beams
}

\section{Sara Bolognesi*}

IRFU, CEA, Université Paris-Saclay, Gif-sur-Yvette, France

E-mail: sara.bolognesi@cea.fr

Neutrino physics with particle beams is bringing the study of neutrino oscillations into the precision era. Latest results from running experiments (NOVA and $\mathrm{T} 2 \mathrm{~K}$ ) provide first hints on Charge-Parity violation and mass hierarchy determination. The next generation of experiments (DUNE and HyperKamiokande) will provide unprecedented statistics and thus will face new challenges in the control of systematic uncertainties. Thanks to their complementarity in detector technology and analysis strategies, the comparison and, eventually, combination of those experiments will provide strong and new ways to constrain such uncertainties. More fundamentally, the comparison of neutrino oscillation measurements at different energies and different baselines will allow to characterize neutrino oscillations in a complete and open-minded approach, beyond the limits imposed by the PMNS paradigm.

*** The European Physical Society Conference on High Energy Physics (EPS-HEP2021), ***

*** 26-30 July $2021 * * *$

*** Online conference, jointly organized by Universität Hamburg and the research center DESY ***

${ }^{*}$ Speaker 


\section{Introduction}

Neutrino physics has a vibrant present and a bright future. Present experiments relying on neutrino production with beams from accelerators (NOVA [1] and T2K [2, 3]) brought neutrino oscillation measurements into the precision era. The FNAL and JPARC laboratories have running accelerators to produce neutrino beams and are developing more powerful beams for the future, while CERN is playing a pivotal role as platform for dedicated detector development. The next-generation of experiments (DUNE [4] and HyperKamiokande [5]) will be world-wide efforts, comparable in complexity and in size to experiments at colliders. This master plan is complemented with a rich program of experiments to measure neutrino-nucleus scattering cross-sections, to search for nonstandard neutrino oscillations and to push further the R\&D for neutrino detectors. Last but not least, a crucial contribution from the nuclear-theory community and from phenomenologists all around the world is laying the ground to enable proper interpretation of the experimental results.

The standard paradigm of neutrino oscillations consists of 3 neutrino mass eigenstates mixed through the PMNS matrix to produce 3 flavour eigenstates. In this model, neutrino oscillations are parametrized by 6 free parameters: 3 mixing angles, 2 differences of mass eigenstates and a phase $\delta_{C P}$ which, if different from 0 or $\pi$, would be a fundamental source of violation of Charge-Parity invariance (CPV) in the lepton sector. Neutrino oscillations are also sensitive to the sign of the 2 mass differences and thus can establish the mass ordering of the 3 mass eigenstates. Such ordering (often referred as mass hierarchy, $\mathrm{MH}$ ) is called "normal", if it mirrors the ordering of the chargedlepton masses (e.g., the lightest mass eigenstate has the largest electron-flavour contribution), or "inverted" otherwise.

Accelerator beams produce fluxes enhanced in muon neutrinos (or muon antineutrinos). Accelerated protons impinge on a target and thus create hadrons (mostly pions and kaons) which decays into charged leptons (mainly muons and muon neutrinos). By focusing hadrons of opposite charge, an enhanced flux of neutrinos or antineutrinos is produced. Neutrino long-baseline experiments exploit such beam-produced neutrinos to measure the disappearance of muon (anti)neutrinos and the appearance of electron (anti)neutrinos. The channel of tau neutrinos appearance is limited by the available neutrino energies (if lower than the tau mass then this channel is forbidden) but it is under study as additional (subleading) channel for the next-generation of experiments. The oscillations are measured by comparing the neutrino rate and energy spectrum, for each flavour, between the near detector(s), placed near the neutrino source before standard oscillation can happen, and the far detector(s), placed at a given distance (baseline). Neutrinos are subject to interaction with the Earth matter along the distance they travel. These "matter effects" can be parametrized as if neutrinos in matter had a different effective mass than neutrinos in vacuum.

In the very rough 2-flavour approximation, the disappearance probability can be written as

$$
1-P\left(v_{\mu} \rightarrow v_{\mu}\right) \sim \sin ^{2}(2 \theta) \sin ^{2}\left(1.27 \frac{\Delta m^{2}\left[e V^{2}\right] L[k m]}{E[G e V]}\right)
$$

The oscillating behavior goes with the ratio of the baseline $(L)$ over the neutrino energy $(E)$ and maximal (dis-)appearance is expected at specific values of such ratio. The flux neutrino energy and the baseline of the experiments are chosen accordingly: for a given baseline, the spectrum of the muon (anti)neutrinos shows a depletion at the energy corresponding to the oscillation maxima. 

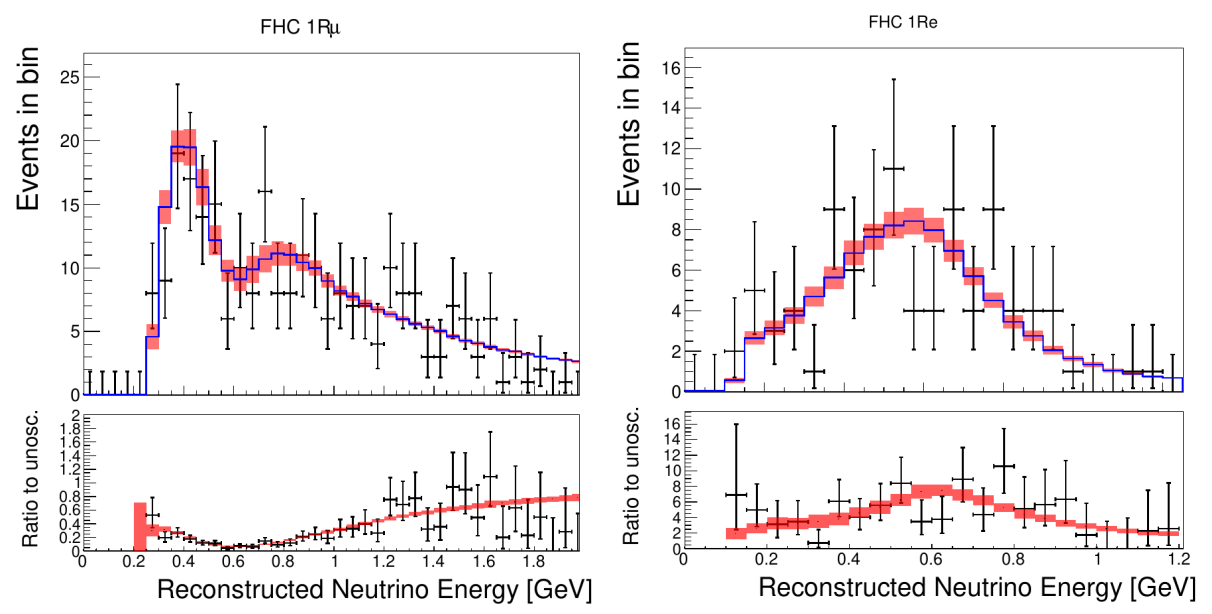

Figure 1: Spectra of muon (left) and electron (right) neutrino candidates at the T2K far detector.

As can be inferred by the equation above, to first order, the mixing angle $(\sin \theta)$ is measured by the amplitude of the depletion (and is thus sensitive to uncertainties on the neutrino rate) and the mass difference $\Delta m^{2}$ is measured by the position of the depletion (and is thus sensitive to uncertainties on the neutrino energy reconstruction). Figure 1 shows, as an example, the T2K muon and electron neutrino spectra at the far detector: at the energy of the oscillation maximum, where muon (anti)neutrinos are depleted, the appearance of electron (anti)neutrinos is observed. Both $\mathrm{CPV}$ and matter effects induce an asymmetry between electron neutrino and electron antineutrino appearance. CPV happens if the oscillation in 'vacuum' is different for neutrinos and antineutrinos. Matter effects are different between neutrinos and antineutrinos, since they rise effectively from the charged-current interaction with the Earth matter, and they depend on the sign of $\Delta m^{2}$ (and thus on the MH). The longer is the baseline, the largest are the matter effects, the strongest is the sensitivity to $\mathrm{MH}$.

\section{Present and next generation of neutrino long-baseline experiments}

Today two long-baseline experiments are measuring neutrino oscillations: NOVA and T2K. Both experiments exploit the off-axis technique: the near and far detectors are placed at a small angle with respect to the beam axis. Because of the two-body decay kinematics ( $\pi \rightarrow \mu v)$, the energy of the neutrinos depends on their angle with respect to the parent $\pi$ direction. Thanks to this technique, T2K and NOVA exploit a narrow-width neutrino energy flux, centered at the oscillation maximum and minimizing the background of electron neutrinos produced in the beamline (which come mostly from kaon and muon three-body decays and thus do not feature the same angle-energy dependence).

NOVA experiment features a neutrino flux peaked at $2 \mathrm{GeV}$ (at the NUMI beamline in Fermilab), a $14 \mathrm{kT}$ far detector, placed on surface at a baseline of $810 \mathrm{~km}$, composed of PVC cells filled with liquid scintillator, and a near detector functionally identical to the far detector. Muon and electron neutrinos are identified through the charged leptons produced by charged-current interactions with the detector target nuclei: muons leave a long, clean track while electrons create a shower. The 
background due to $\pi^{0}$ produced in neutral-current interactions is rejected through topological cuts relying on the separation of their two decay gammas.

T2K experiment features a neutrino flux peaked at $600 \mathrm{MeV}$ (at JPARC beamline), SuperKamiokande as far detector, a $50 \mathrm{kT}$ water-Cherenkov detector placed deep underground at a baseline of $295 \mathrm{~km}$, and a near detector suite including multiple detectors. INGRID [6] and MUMON [7] monitor the stability of the beam direction and position. ND280 (planned to be upgraded [8] in 2022) is a magnetized detector allowing to measure separately neutrinos and antineutrinos through their interactions with carbon and water targets. Finally WAGASCI-BabyMIND $[9,10]$ is a magnetized detector with enhanced water target and placed at a different off-axis angle to control the neutrino-nucleus cross-section as a function of nuclear target and neutrino energy. The SuperKamiokande far detector identify muons and electrons through the shape of their Cherencov rings and rejects $\pi^{0}$ background by reconstructing the two rings from its decay gammas.

The main NOVA and T2K results in the electron (anti)neutrino appearance channels are summarized in Fig. 2. The long NOVA baseline allows a good sensitivity on the MH but present results lie in the region of degeneracies between different $\mathrm{MH}$ possibilities and different values of $\delta_{C P}$. T2K results are minimally sensitive to $\mathrm{MH}$ and feature a clean sensitivity to $\delta_{C P}$, in particular present results indicate a preference for maximal CPV (CP-conservation being disfavoured at $90 \%$ confidence level). The most recent measurements of $\left|\Delta m_{32}^{2}\right|$ and $\sin \theta_{23}$, dominated by the muon(anti)neutrino disappearance channel, can be found in $\operatorname{Ref}[11,12]$.

A steady program of beam upgrades has started for T2K [13]: from $500 \mathrm{~kW}$ to $750 \mathrm{~kW}$ in 2022, and then steadily increasing up to $1.3 \mathrm{MW}$ at the start of HyperKamiokande. T2K will exploit, until HyperKamiokande starting date, the upgraded beam in a new phase of data taking with the upgrade of ND280 and Gadolinium doping at SuperKamiokande. T2K, by itself, features a $3 \sigma$ sensitivity in case of maximal CPV. HyperKamiokande, planned to start in 2027, will exploit a gigantic water-Cherencov far detector, more than 8 times larger than SuperKamiokande. The detector will be installed deep underground at the same off-axis angle as SuperKamiokande but in the opposite direction. ND280 upgrade will be ported from T2K to HyperKamiokande enabling a robust anchoring point to control the beam and the neutrino interactions, which are the main source of systematic uncertainties for neutrino oscillation measurements. HyperKamiokande features a very large sensitivity to CPV and a very fast discovery potential, in case of maximal violation, as shown in Fig. 3. Such large sensitivity is directly due to the huge statistics enabled by the HyperKamiokande beam and far detector. The robustness of such expectations are granted by the steady program of $\mathrm{T} 2 \mathrm{~K}$ data taking to characterize the upgraded neutrino beam and to improve the control the systematic uncertainties to cope with such statistical precision. Given the relatively short baseline, same as $\mathrm{T} 2 \mathrm{~K}$, the $\mathrm{MH}$ will be measured with atmospheric neutrinos as shown in Fig. 3.

The other next-generation experiment in preparation is DUNE, which will exploit a new neutrino beam under development at Fermilab, planned to start with $1.2 \mathrm{MW}$ and to ultimately reach 2.4 MW. Such beam will produce a neutrino flux spanning 1-4 GeV in energy (wide-band). DUNE will exploit four large Liquid-Argon (LAr) Time Projection Chambers (TPC) of about $10 \mathrm{kTon}$ fiducial mass each, placed at a baseline of $1300 \mathrm{~km}$ and installed with a staged approach until 2035. The very large baseline allows extremely fast MH determination, as shown in Fig. 4, while $5 \sigma$ sensitivity on CPV could be reached after the beam upgrade to $2.4 \mathrm{MW}$ : in the 5 first 

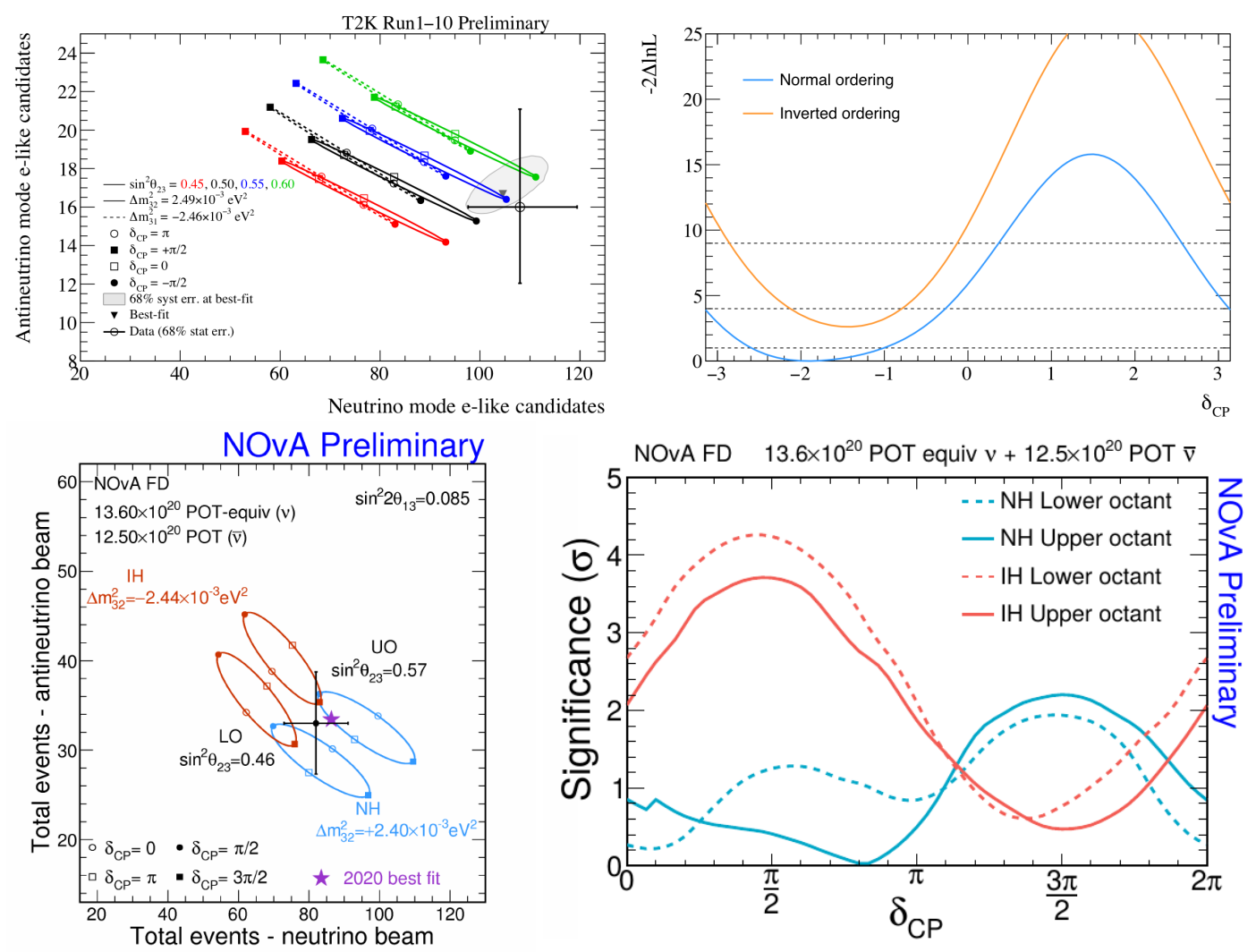

Figure 2: Latest T2K (top) and NOVA (bottom) results in the electron (anti)neutrino appearance (left) and analysis likelihoods as a function of $\delta_{C P}$ (right).

years of the experiment a sensitivity around $3 \sigma$ on CPV is expected. The novelty of DUNE reside on the wide-band beam, covering two oscillation maxima, and on the LAr technology, enabling to reconstruct in full details the final state particles produced by neutrino interactions, thus measuring with very good resolution the neutrino energy. The combination of such features will provide extremely rich information on the oscillation shape as a function of energy, to be exploited for precision measurements of the oscillation parameters. At the same time, new challenges arise related with the control of systematic uncertainties on a new neutrino beam, on a (relatively) new detector technology and on a nuclear target (argon) less explored and more complex than carbon or oxygen. The importance of such aspects and the plan to master them, for both the next-generation experiments, will be highlighted in the next section.

\section{The challenges ahead: the impact of systematic uncertainties and the role of near detectors}

The experiments of next generation will enjoy thousands of electron (anti)neutrinos in the appearance channel and more than ten thousand muon (anti)neutrinos in the disappearance channels. Accordingly, the systematics uncertainties due to the modeling of neutrino flux, neutrino interactions and near and far detectors will have to be controlled at unprecedented level. For CPV discovery and $\mathrm{MH}$ determination the most impactful systematic uncertainty are the ones affecting the rate of 

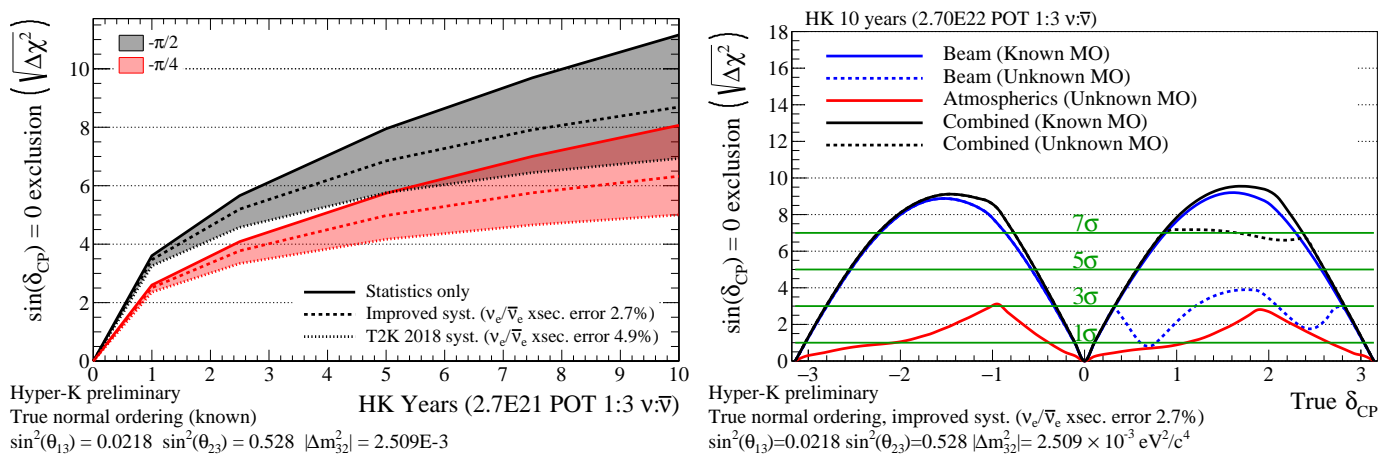

Figure 3: HyperKamiokande sensitivity to CPV discovery as a function of time (left), CPV sensitivity using beam and atmospheric neutrinos in case of unknown MH (right).
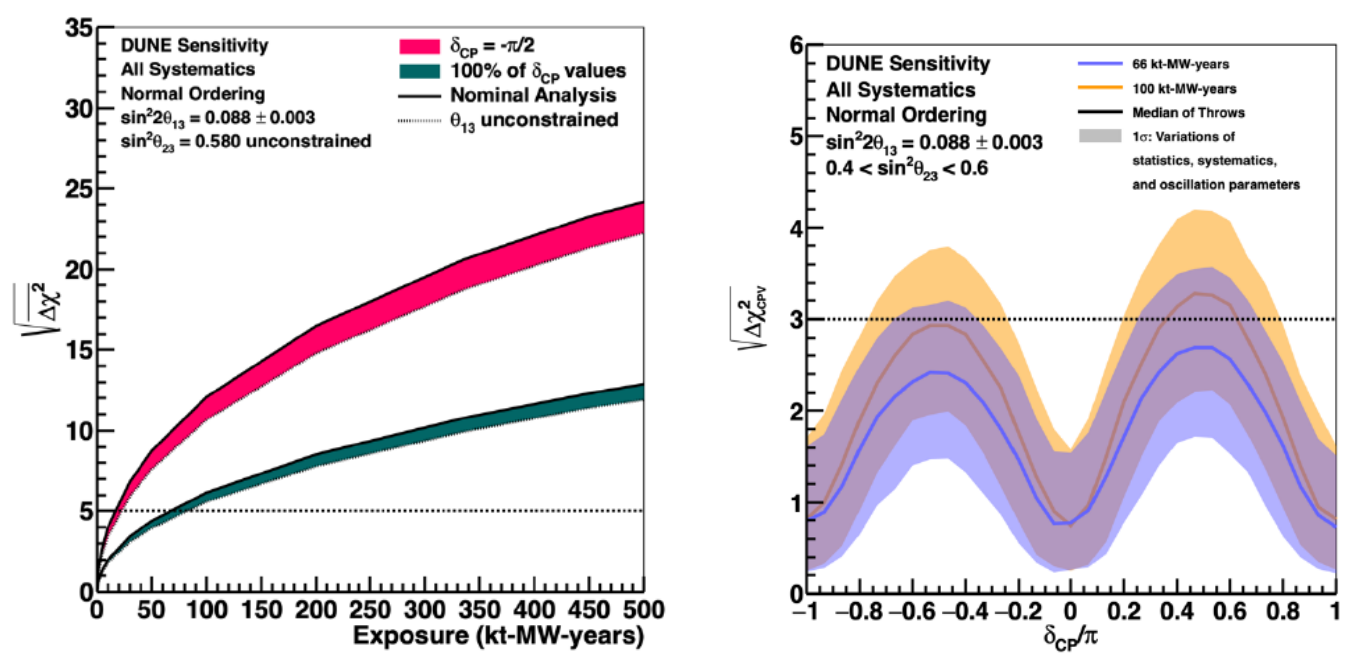

Figure 4: DUNE MH sensitivity as a function of exposure (left), CPV sensitivity for the early data-taking scenarios (right).

electron neutrino and antineutrino (and in particular the anticorrelations between the two rates). For precision measurements the accuracy of neutrino energy reconstruction is crucial. For instance, to meet the precision target of 15 degrees on $\delta_{C P}$ and $1 \%$ on $\Delta m_{32}^{2}$ measurements, a control of energy scale better than $1 \%$ is needed, including the knowledge of flux energy peak, the corrections due to nuclear effects (e.g., nuclear removal energy) and the detector calibration. The present experiments (T2K, NOVA) are opening the road indicating analysis strategies and detector designs enabling such precision. In particular, a new generation of near detectors is being developed to this aim.

The measurement of the neutrino rate as a function of energy at the near detector is the convolution of neutrino flux produced at the accelerator and the neutrino-nucleus cross-section. At the far detector those are further convoluted with the neutrino oscillation probability, which is the target of the measurement. Near detectors are thus used to measure flux and cross-sections. Depending on the experiment, the extrapolation of the near detector constraints to the far detector can be affected by differences in acceptances and nuclear targets but, actually, the most complex challenge, which is common to all experiments, rises from two facts: the neutrino energy distribution 
is different before oscillation (as measured at near detectors) and after oscillation (as measured at far detectors) and the near detector measures the convolution of flux and cross-section, while those must be disentangled in order to be propagated separately to the far detector. Because of those reasons, the extrapolation of the near detector constrains to the far detector always relies on some assumptions from nuclear models for flux production and neutrino-nucleus cross-section.

Very sophisticated nuclear models are needed to model the productions of hadrons from protons impinging on the beamline target, such hadrons then interact further with the target itself and with the material in the beamline and finally decay to produce the neutrino flux. Dedicated experiments (like NA61/SHINE [14]) are performed to measure the produced hadron multiplicity and kinematics and tune the predictions from nuclear models. For instance, a recent measurement from NA61/SHINE [15] on a target replicating exactly the T2K target allowed to strongly reduce the uncertainty on the T2K flux modeling (at $5 \%$ up to $4 \mathrm{GeV}$ ).

Very sophisticated nuclear models are also needed for neutrino-nucleus interactions in order to extrapolate the near detector measurements, at the source flux energy, to the oscillated flux at the far detector. The impact of modelling on such extrapolation get strongly reduced if the cross-section can be precisely measured as a function of energy at the near detector. But the neutrino energy is not a direct observable, it is known on-average by the flux modeling, but it can only be reconstructed event-by-event through the final state particles produced by the neutrino interaction.

In order to improve the precision of neutrino energy reconstruction event-by-event, and thus minimizing the systematic uncertainty in the extrapolation from the near to the far detector, a new design of near detector has been developed by T2K: the upgrade of ND280 [8]. A new scintillating target composed of multiple $1 \mathrm{~cm}^{3}$ cubes, readout by fibers in the 3 directions, allows to reconstruct all the particles produced in the interactions, including low momentum pions and protons. Moreover, for the first time, neutrons can be reconstructed by measuring the time of flight between the neutrino vertex and isolated clusters, corresponding to neutrons re-interactions in the detector. The new target is surrounded by TPCs to track and identify particles escaping the target. In particular two new TPCs are being developed to be placed on the top and on the bottom of the target to maximize the angular acceptance. These new TPCs are based on the resistive Micromegas technology to optimize the momentum resolution of charged particles and thus improve the precision of neutrino energy reconstruction.

The design of this detector is based on the seminal work of ND280 [16, 17] and MINERvA $[18,19]$ which have shown the importance to measure exclusive final states in order to constrain uncertainties due to nuclear physics. The capabilities of the ND280 upgrade detector in this respect has been quantified for T2K and HyperKamiokande statistics in Ref. [20]. This near detector will allow for the first time to reconstruct completely the final state particles from neutrino interactions and thus measure the neutrino energy with unprecedented precision. In particular, the importance of having neutrons measured for the first time is crucial: for instance, in DUNE, the impact of 'missing energy' in the reconstructed neutrino energy is one of the largest and most difficult issues to control (as shown in Ref. [21]).

An alternative approach is also being developed for the next-generation of experiments, based on the idea of 'movable' near detectors along the off-axis angle. The concept of a water Cherenkov near detector movable vertically has been proposed for HyperKamiokande in Ref. [22]. Similarly, a LAr TPC movable horizontally is the primary near detector for DUNE [23]. Given the dependence 
of the neutrino energy with the off-axis angle, mentioned above, the capability of spanning different off-axis angle will give access to a measurement of flux and cross-section as a function of neutrino energy. In this case the energy is not necessarily known precisely event-by-event but on average over 'bins' of flux energy whose width is determined by the capability of collecting large enough statistics at many slightly different off-axis angles. In this approach the cancellation of cross-section uncertainty is done at the expense of flux uncertainties, notably the flux must be extremely well under control as a function of the angle and of time. The cancellation of cross-section uncertainties is here achieved in an 'effective' way: the understanding and correct modeling of nuclear effects in neutrino energy reconstruction can be overcome, as long as, the oscillated energy spectrum can be reproduced precisely by combining energy spectra collected at the far detector from different off-axis angles. The precision of this approach is limited by the amount of statistics collected at each off-axis angle and by the beam stability over time. It is clear that combining this approach with precise neutrino energy reconstruction at the near detectors would further enhance the capability of making precise predictions of neutrino oscillated rate and spectrum at the far detector for the precise measurement of oscillation probabilities.

\section{Comprehensive, precise and open-minded characterization of neutrino oscillations}

The definitive establishment of CPV, especially in presence of large CPV as suggested by present results, and of $\mathrm{MH}$, could be considered 'low-hanging fruits' for the next generation of oscillation experiments exploiting beams from accelerators. Beyond that, the precision measurements of PMNS mixing parameters will pose unprecedented challenges on the control of systematic uncertainties related with flux, neutrino-nucleus cross-section and detector response modeling. To reach this aim, the combination of DUNE and HyperKamiokande will give invaluable inputs. In precision measurements which will be dominated by systematic uncertainties, cross-checking results between experiments with different detector technology would give strong confidence on the control of detector modelling (eg, energy scale, particle identification for background suppression, ....). While building a comprehensive neutrino-nucleus cross-section model at different targets and energies is a huge challenge, the measurement of neutrino-nucleus cross-sections in the different near detectors of the two experiments will help cross-checking the validity of the corresponding systematic model. Measurements at different energies and targets are a powerful leverage to challenge and improve the models. For instance different interaction channels are known to have different A-dependence on the nucleus size and such feature could be used to disentangle them when direct, separate measurements are not accessible otherwise experimentally. A practical example is the fraction of events with pion(s) and proton(s) produced at the neutrino interaction vertex but reabsorbed into the nucleus (and thus not observable experimentally). In such events, the neutrino energy could be incorrectly estimated, unless a correction is applied on the basis of the nuclear model. The fraction of such events is different for different nuclear targets, thus comparing measurements of pion and proton multiplicity on different nuclei may help evaluating the rate of events affected by hadron reabsorption.

From a more general standpoint, present results are strongly constrained by the PMNS paradigm which is quite strict and not (yet?) motivated by fundamental symmetries. The precision measure- 
ments will help cornering down such possible symmetries. Even more importantly, the combination of experiments at different $\mathrm{L} / \mathrm{E}$ will allow to characterize neutrino flavour mixing in a more general paradigm. Quite strong assumptions like the minimal-3 flavour scenario, the existence of only SM neutrino interactions for production and detection and, correspondingly, of only 'standard' matter effects along the propagation, should be released for a complete, open-minded interpretation of oscillation data. The combination of experiments at different $\mathrm{L} / \mathrm{E}$ will increase the sensitivity to BSM scenarios well beyond the simple 'sum of the likelihoods', it will allow to avoid degeneracies between BSM effects and standard PMNS effects: in some cases, BSM effects could even obfuscate PMNS effects and induce wrong interpretation of the data. For instance, new CPV sources in Non Standard Interactions (NSI) could modify the electron neutrino - antineutrino appearance asymmetry. Asymmetry values compatible with no CPV in the PMNS paradigm could result from the conspiracy of 'standard' CPV with specific values of CPV from NSI. As shown for instance in Ref. [24], studying oscillations at different energies for the same baseline could help solve such degeneracies. A further step into this investigation, consists in the attempt of characterizing the existence of CPV in the most model-independent way, as explained in Ref. [25]. The approach consists in searching for T-violation (the mirror of $\mathrm{CPV}$ ) by looking at experiments at different baselines and same energy allowing for arbitrary (non-standard) matter effect, for arbitrary (non-unitary) mixing between flavour and mass eigenstates (even different for production and detection).

For such type of model-independent studies, as well as for precision measurements in the PMNS paradigm, a precise and unbiased neutrino energy reconstruction is needed. Also in this respect, the comparison of HyperKamiokande and DUNE will be crucial. The two experiments, indeed, reconstruct the neutrino energy in very different ways. The LAr technology of DUNE is designed to make an exclusive measurement of the final state of neutrino interactions by detecting most of the particle in the final state (possibly complemented by visible energy deposits around the vertex due to low momentum particles and with the major exception of neutrons which are extremely hard to detect). HyperKamiokande, on the other hand, relies on leptons and pions above Cherencov threshold (protons are most of the time below such threshold). At the HyperKamiokande energy, pion production is suppressed and neutrino energy could be evaluated relying solely on lepton kinematics for most of the events, assuming Quasi-Elastic scattering. Many nuclear effects will impact both energy reconstruction strategies (binding energy, Fermi momentum, hadron nuclear reabsorption, ...) but in different ways. A coherent comparison of the results of the two experiments would provide strong confidence on the correctness of the modeling of such nuclear effects and thus on an unbiased neutrino energy reconstruction. Clearly, as previously mentioned, also a very good control of detector modeling is needed in parallel to avoid biases on neutrino energy reconstruction.

\section{Summary}

The T2K oscillation measurement made the cover of Nature in April 2020 with first $3 \sigma$ limits on the value of $\delta_{C P}$ : such results are still limited by statistics. Both T2K and NOVA will keep taking data in next years, thus the next future of the domain is very promising. The next-generation experiments (DUNE and HyperKamioaknde) are worldwide efforts comparable to experiments at colliders. They will enable unprecedented statistics (more than 20 times larger than today available) and thus they will need an unprecedented control of systematics uncertainties (at the level of 1\%). 
T2K and NOVA are opening the road to exercise new near detectors, new analyses techniques and participating to the complex work of improving the modeling of nuclear effects in collaboration with the worldwide nuclear theory community. An intensive work of detector development (R\&D, prototypes, test beams) is on-going at the CERN Neutrino Platform to prepare the detectors of the next-generation of experiments. A vibrant community ready to react to the 'unexpected' is under formation: new (today negligible) systematic uncertainties and/or BSM signs are expected in the future. An inventive usage of near detectors and the exploration of complementarity between HK and DUNE are the most important resources in our hands for a robust strategy to address future 'unknown unknowns'. More in general, the study of oscillations should aim to go beyond the strict, 'accidental' PMNS paradigm and characterize neutrino mixing with open mind, combining experiments at different energies and baselines.

\section{References}

[1] M. A. Acero et al. (NOvA) (2021), 2109.12220.

[2] K. Abe et al. (T2K), Nucl. Instrum. Meth. A 659, 106 (2011), 1106. 1238.

[3] K. Abe et al. (T2K), Nature 580, 339 (2020), [Erratum: Nature 583, E16 (2020)], 1910 . 03887.

[4] B. Abi et al. (DUNE), JINST 15, T08008 (2020), 2002.02967.

[5] K. Abe et al. (Hyper-Kamiokande) (2018), 1805.04163.

[6] K. Abe et al., Nucl. Instrum. Meth. A 694, 211 (2012), 1111 . 3119.

[7] K. Suzuki et al. (T2K), PTEP 2015, 053 C01 (2015), 1412. 0194.

[8] K. Abe et al. (T2K) (2019), 1901.03750.

[9] T. Koga et al., JPS Conf. Proc. 8, 023003 (2015).

[10] M. Antonova et al., in Prospects in Neutrino Physics (2017), 1704.08079.

[11] M. A. Acero et al. (NOvA) (2021), 2108. 08219.

[12] P. Dunne (2020), URL https: //doi .org/10.5281/zenodo. 3959558.

[13] K. Abe et al. (T2K, J-PARC Neutrino Facility Group) (2019), 1908. 05141.

[14] N. Abgrall et al. (NA61/SHINE), Eur. Phys. J. C 76, 84 (2016), 1510.02703.

[15] A. Acharya et al. (NA61/SHINE), Phys. Rev. D 103, 012006 (2021), 2010 . 11819.

[16] K. Abe et al. (T2K), Phys. Rev. D 98, 032003 (2018), 1802 . 05078.

[17] K. Abe et al. (T2K), Phys. Rev. D 103, 112009 (2021), 2102 . 03346.

[18] M. Elkins et al. (MINERvA), Phys. Rev. D 100, 052002 (2019), 1901.04892.

[19] T. Cai et al. (MINERvA), Phys. Rev. D 101, 092001 (2020), 1910.08658.

[20] S. Dolan et al. (2021), 2108.11779.

[21] A. M. Ankowski and C. Mariani, J. Phys. G 44, 054001 (2017), 1609. 00258.

[22] S. Bhadra et al. (nuPRISM) (2014), 1412 . 3086.

[23] A. A. Abud et al. (DUNE ), Instruments 5, 31 (2021).

[24] J. M. Hyde, Nucl. Phys. B 949, 114804 (2019), 1806.09221.

[25] T. Schwetz and A. Segarra (2021), 2106. 16099. 